Revista de Psicología de la PUCP. Vol. XXII, 2, 2004

\title{
Análisis factorial confirmatorio de la Escala de Estilos de Crianza de Steinberg: validez preliminar de constructo
}

\author{
César Merino Soto ${ }^{1}$ \\ Defensoría Municipal del Niño y \\ del Adolescente
}

\author{
Stephan Arndt ${ }^{2}$ \\ Iowa Consortium for Substance Abuse, \\ Research and Evaluation
}

Se estudió la validez de constructo y confiabilidad interna de la Escala de Estilos de Crianza de L. Steinberg que se administró a una muestra de 224 adolescentes entre 11 y 19 años de un colegio público en Lima. Mediante el análisis factorial confirmatorio de grupo múltiple, la estructura de tres subescalas (Compromiso, Autonomía Psicológica y Control Conductual/Supervisión) se mantuvo en general estable, aunque la agrupación teórica de los ítems tendió a estar influenciada por la distribución de los ítems. La confiabilidad alfa para las subescalas solo alcanzó niveles marginales de aceptación y se reconoció el probable impacto del error de medición aleatorio. Los resultados muestran que el instrumento utilizado es una herramienta potencial para la investigación, pero estos hallazgos son preliminares y se requieren estudios adicionales para evaluar la generalización del análisis efectuado.

Palabras clave: análisis factorial confirmatorio, estilos de crianza, adolescentes.

\section{Confirmatory factorial analysis of Steinberg's Parenting Style Scale: Preliminary construct validity}

The present study tried to find evidences of construct validity and internal reliability for the Parenting Styles Scale of $L$. Steinberg. The instrument was applied in a sample of 224 adolescents from 11 to 19 years old from a public school of Lima. With a strategy of multiple group confirmatory factor analysis, the three-subscale structure (Commitment, Psychological Autonomy and Behavior Control/Supervision) was in general stable, although the theoretical grouping of the items was influenced by the distribution of the item. The alpha reliability reached marginal levels and the probable impact of the random error was recognized. Results show that this instrument is a potential tool for research, but these findings are preliminary and additional studies are required to evaluate the generalization of the analysis.

Key words: confirmatory factor analysis, parenting styles, adolescents.

Psicólogo, graduado en la Universidad Inca Garcilaso de la Vega, Perú. Responsable del Servicio de Psicología de la DEMUNA, Chorrillos. Presta asistencia en la intervención y prevención del maltrato intrafamiliar y escolar. Sus investigaciones se orientan hacia la metodología psicométrica, la crianza y la evaluación psicológica. Correo electrónico: sikayax@yahoo.com.ar 

Desde la antigüedad, la relación padre-hijo ha sido reconocida como un eje crítico para la salud mental de ambos y para las direcciones que toma la influencia dominante del padre sobre los hijos. A través de los años ha proliferado la investigación sociológica y psicológica centrada en la dinámica intra y extrafamiliar para explicar y predecir los resultados conductuales en las siguientes generaciones, a través la crianza. En uno de los campos más prolíferos de la psicología, esto es la medición psicológica, la investigación de la crianza ha avanzado un paso importante con la construcción de instrumentos de medición que describan los estilos de crianza definidos por la teoría.

Se ha reconocido que los problemas de la crianza son mayormente predictores lineales del desajuste en los sujetos. Por ejemplo, las investigaciones de Lieb y colaboradores han demostrado las relaciones entre variables de la relación padre-hijo y cuadros psicopatológicos tal como han sido definidos por el Diagnostic and Statistical Manual of Mental Disorders-IV (APA, 1994). Así, se tiene la relación entre la historia parental de alcoholismo con la progresión del uso del alcohol en los descendientes (Lieb et al., 2002), estilos de crianza y fobia social (Lieb et al., 2000) y depresión parental y emergencia de desórdenes depresivos (Lieb, Isensee, Höfler, Pfister \& Wittchen, 2002; Lieb, Isensee, Höfler \& Wittchen, 2002). Sin embargo, parece que la elaboración y evaluación psicométricas de los

2 Doctor en Psicología. Profesor de psiquiatría en la Escuela de Medicina y de bioestadística en la Escuela de Salud Pública, Universidad de Iowa. Director del Iowa Consortium for Substance Abuse Research and Evaluation. Correo electrónico: stephan@avalon.net 
instrumentos para explorar la crianza en el medio latinoamericano no han sido favorecidas frecuentemente por este reconocimiento.

Un estudio que explore las características psicométricas de una prueba seleccionada debería abarcar los aspectos básicos sobre la información para que el usuario, por ejemplo psicólogos escolares y clínicos, juzgue su calidad métrica relacionada con lo que se pretende medir. A diferencia de la medición física, la medición psicológica contiene mayor varianza de error, lo que origina que la planificación de estudios de validez y confiabilidad sea una norma más que una excepción cuando se quiere elegir el instrumento para un nuevo contexto de aplicación. Esto es aún más crítico en la investigación intercultural (cross-cultural), pues no se debe asumir a priori que el constructo medido con una prueba en una cultura específica es transportable directamente a otra (Navas, 2001).

En la investigación sobre la crianza, esta necesidad ha sido satisfecha en algunos estudios dedicados a la generalización de aspectos definidos de la crianza en num rosos países (Arrindel et al., 2001). Por otro lado, como los índices psicométricos que se extraen desde la teoría clásica de los test dependen de la muestra estudiada, en los estudios psicométricos también se considera si las submuestras introducen variabilidad en parámetros estadísticos (Gregory, 2000). Por ejemplo, la confiabilidad es dependiente de la muestra en que se aplica una prueba y debe ser examinada en cada muestra (Nunnally \& Bernstein, 1995).

\section{Estilos versus Prácticas de Crianza}

La mayoría de los investigadores que intentan describir los estilos de crianza recurren al concepto elaborado por Baumrind (Darling, 1999). El constructo de estilo de crianza captura las variaciones normales en las estrategias de enseñanza, socialización y control hacia los niños (Baumrind, 1991).

Darling y Steinberg (1993) definieron el estilo de crianza como "una constelación de actitudes hacia los niños, que son 
comunicadas hacia él y que, tomadas en conjunto, crean un clima emocional en que se expresan las conductas de los padres (1993, p. 488). El estilo de crianza, por lo tanto, está bien asociado con el clima emocional que sirve como fondo de las interacciones padre-niño. Este concepto es esencialmente global y está en un nivel de abstracción por sobre otras definiciones provenientes de aspectos más específicos de la crianza. Desde la investigación empírica y los modelos de crianza informados en la literatura, uno de estos aspectos más específicos es el de prácticas de crianza están dirigidas por ciertas metas, tales como la estimulación del logro académico o metas de socialización (Darling \& Toyokawa, 1997).

Las escalas construidas para evaluar la crianza generalmente han tendido a confundir el estilo de crianza con las prácticas de crianza (Darling \& Toyokawa, 1997), así como estilos de crianza y dimensiones de crianza (por ejemplo, Steinberg, Lamborn, Dornbusch \& Darling, 1992).

Algunas evalúan directamente los estilos de crianza, como el elaborado por Buri (1991), y otros exploran dimensiones dependientes de estos estilos globales y que son extraídos empíricamente (Perris, Jacobsson, Lindström, von Knorring \& Perris, 1980; Robinson, Mandleco, Olsen \& Hart, 1995).

\section{Los modelos de Baumrind y de Maccoby y Martin}

Maccoby y Martin (1983) propusieron una clasificación bidimensional de los patrones de crianza, tal como está representado en el Cuadro 1. 
César Merino Soto y Stephan Arndt

\section{Cuadro 1}

Tipología de patrones de crianza según Maccoby y Martín (1983)

\begin{tabular}{ccc}
\hline & $\begin{array}{c}\text { Centrado en el niño, } \\
\text { aceptante, sensible }\end{array}$ & $\begin{array}{c}\text { Centrado en el padre, } \\
\text { rechazante, insensible }\end{array}$ \\
\hline $\begin{array}{c}\text { Demandante, } \\
\text { controlador }\end{array}$ & $\begin{array}{c}\text { Autoritativo, } \\
\text { recíproco, } \\
\text { comunicación altamente } \\
\text { bidireccional }\end{array}$ & $\begin{array}{c}\text { Autoritario, } \\
\text { de control, } \\
\text { no exigente }\end{array}$ \\
Indulgente, & dominio a través de poder \\
& muy flexible & $\begin{array}{c}\text { Negligente, } \\
\text { indiferente, }\end{array}$ \\
\hline
\end{tabular}

Según estos autores, utilizando estos dos ejes ortogonales, es decir, el eje del control y de lo afectivo-actitudinal, se pueden identificar cuatro patrones. Similar a lo anterior, Schludermann y Schludermann (1988) agruparon en tres dimensiones ortogonales todos los factores involucrados en la exploración de los diferentes factores en la crianza (por ejemplo, intrusividad, aceptación, posesividad, control, coerción, inconsistencia, extrema autonomía, etc.): aceptación-rechazo, control psicológico-autonomía psicológica y control indulgente-control firme. En general, muchos de los componentes de la crianza deben ser vistos a lo largo de un continuo, en el que los factores ubicados en sus antípodas, definen los extremos del control, del afecto, de la autonomía y del elogio que los padres aplican.

Los estilos propuestos por Maccoby y Martin son una extensión de las elaboraciones de Baumrind, y parece que estos estilos pueden hallarse en las familias con adolescentes. Se ha reportado que durante la adolescencia se pueden identificar tres componentes de los estilos de crianza están sustentados teórica y empíricamente. Estos son el control conductual, la responsividad parental y la autonomía psicológica (Steinberg, Elman \& Mounts, 1989). 
Análisis factorial confirmatorio de la Escala de Estilos de Crianza de Steinberg

Los padres autoritativos se caracterizan por ser orientados racionalmente, exigentes con las normas, cálidos escuchan a los hijos, se desenvuelven en una relación dar-tomar, mantienen altas expectativas, son afectuosos, monitorean activamente la conducta sus hijos, y les proveen de estándares de conducta en un contexto de relaciones asertivas, más que restrictiva o intrusivamente.

Los padres autoritarios son aquellos que imponen normas rígidas, afirman su poder sin cuestionamiento, usan la fuerza física como coerción o como castigo y a menudo, no ofrecen el cariño típico de los padres autoritativos. Son altamente exigentes, demandantes y directivos, y muestran bajos niveles de expresiones afectivas. Están orientados hacia la afirmación del poder y la búsqueda de la obediencia; llegan a ser altamente intrusivos.

Los padres permisivos son aquellos que permiten que los hijos regulen sus propias actividades con relativamente poca interferencia. Generalmente no imponen reglas; los hijos hacen sus propias decisiones sin consultar usualmente a los padres. Típicamente son cariñosos y bondadosos, explican las cosas usando la razón y la persuasión más que la afirmación de poder. Los hijos tienen más probabilidad de presentar problemas académicos y de conducta. Estos padres son llamados también, padres indulgentes o no directivos. Puntúan más alto en afectividad/responsividad y bajo en exigencia/disciplina. Permiten la auto-regulación del propio niño, lo que los lleva a estimular la independencia y el control bajo las propias creencias y necesidades. En los encuentros disciplinarios, tienden a evitar la confrontación y generalmente ceden a las demandas de los hijos.

Los padres negligentes son aquellos que muestran poco o ningún compromiso con su rol de padres. No ponen límites a sus hijos porque no hay un verdadero interés por hacerlo. Les faltan respuestas afectivas o de control conductual en situaciones diarias y/o en aquellas en que críticamente se requieren. Son padres que puntúan bajo en las dos dimensiones de exigencia y afectividad. En casos extremos, son explícitamente rechazantes. 
Basado en lo anterior planteamos la pregunta del presente estudio de investigación: ¿cuáles son las características psicométricas de validez y confiabilidad de la Escala de Estilos de Crianza de L. Steinberg, respecto a la validez de constructo y confiabilidad, en una muestra de escolares de 11 a 19 años en un colegiodistrito de Lima? Los objetivos sonque perseguimos aquí serán la obtención de la validez de constructo y confiabilidad interna del instrumento en cuestión. Desde un ángulo exploratorio, observaremos el comportamiento métrico en una muestra de adolescentes peruanos, pero desde un ángulo confirmatorio, evaluaremos lasu asumida estructura interna.

\section{Metodología}

\section{Participantes}

La obtención de la muestra seguirá un muestreo no probabilístico, en que la aleatorización no estará presente en la selección de las unidades de investigación. Este tipo de selección de la muestra es regularmente utilizada en muchas investigaciones sociales y conductuales, nacionales y extranjeras; y tiende a ser suficiente para propósitos exploratorios.

Los participantes fueron estudiantes de secundaria de ambos sexos de un colegio estatal mixto, en el turno mañana y tarde. El rango de edad es de los 11 años a los 19 años, y los grados escolares van desde el ler. año hasta el 5to. año de secundaria. No se observó o recibió alguna información sobre la presencia de alumnos con discapacidades físicas, mentales, cognitivas o neurológicas observables.

El colegio está ubicado en la zona urbana de un distrito costero de Lima. Los estudiantes que asisten a tal colegio provienen de zonas urbanas y peri-urbanas, y provienen de un nivel socioeconómico bajo. 
Análisis factorial confirmatorio de la Escala de Estilos de Crianza de Steinberg

La muestra quedará definida de la manera como aparece en el Cuadro 2.

\section{Cuadro 2}

Información demográfica de la muestra estudiada

\begin{tabular}{|c|c|c|c|c|c|c|}
\hline & \multicolumn{2}{|c|}{11 a 14 años } & \multicolumn{2}{|c|}{15 a 19 años } & \multicolumn{2}{|c|}{ Total } \\
\hline & $n$ & $\%$ & $n$ & $\%$ & $N$ & $\%$ \\
\hline Hombre & 79 & $69.9 \%$ & 73 & $67.0 \%$ & 152 & $68.4 \%$ \\
\hline Mujer & 34 & $30.1 \%$ & 36 & $33.0 \%$ & 70 & 31.9 \\
\hline Total & 113 & $100.0 \%$ & 109 & $100.0 \%$ & 222 & \\
\hline
\end{tabular}

\section{Instrumento}

Se utilizó la Escala de Estilos de Crianza desarrollada por Lawrence Steinberg (Lamborn, Mounts, Steinberg \& Dornbusch, 1991), con la que se examinó los patrones de competencia y ajuste en adolescentes, y sus relaciones con los estilos de crianza.

El instrumento consta de 26 ítems agrupados en tres clusters que definen los aspectos principales de la crianza en adolescentes: compromiso, autonomía psicológica y control conductual. Las dos primeras escalas constan de ítems de 4 opciones, desde 1 (Muy en desacuerdo) a 4 (Muy de acuerdo). La subescala de Compromiso evalúa el grado en que el adolescente percibe conductas de acercamiento emocional, sensibilidad e interés provenientes de sus padres. La subescala de Autonomía Psicológica evalúa el grado en que los padres emplean estrategias democráticas, nocoercitivas y animan a la individualidad y autonomía. La subescala de Control Conductual consta de dos ítems de 7 opciones y otros seis de 3 opciones, y evalúa el grado en que el padre es percibido como controlador o supervisor del comportamiento del adolescente. 
En los estudios de Steinberg y colaboradores (Lamborn, Mounts, Steinberg \& Dornbusch, 1991; Steinberg et al., 1989; Steinberg et al., 1992) el número de ítems fue modificado algunas veces. En Lamborn et al. (1991) se reporta un coeficiente alfa de .72 para Compromiso (10 ítems), y .76 para Control Conductual (9 ítems). En Steinberg et al. (1992), se reporta un coeficiente alfa de .72 (15 ítems), 76 para Control Conductual (9 ítems) y .86 para Autonomía Psicológica (12 ítems). La versión utilizada el presente estudio es la versión definitiva y ha sido utilizada recientemente en un estudio inter-cultural de Mantizicopoulus y Oh-Wang (1998), encontrando coeficientes alfa de $.82, .73$ y .69 en una muestra de adolescentes norteamericanos y $.66, .72$ y .61 en adolescentes coreanos, para Compromiso, Control Conductual y Autonomía Psicológica respectivamente.

Para la construcción de los estilos de crianza basados en los puntajes de las tres subescalas, se siguió el siguiente algoritmo (Cuadro 3).

\section{Cuadro 3}

Construcción de los estilos de crianza basada en los puntajes promedio en las subescalas

\begin{tabular}{lcll}
\hline Estilo de Crianza & Compromiso & Control Conductual & Autonomía Psicológica \\
\hline Padres Autoritativos & Encima del Promedio & Encima del Promedio & Encima del Promedio \\
Padres Negligentes & Debajo del Promedio & Debajo del Promedio & \\
Padres Autoritarios & Debajo del Promedio & Encima del Promedio & \\
Padres Permisivos & & & \\
Indulgentes & Encima del Promedio & Debajo del Promedio & \\
Padres Mixtos & Encima del Promedio & Encima del Promedio & Debajo del Promedio \\
\hline
\end{tabular}

\section{Procedimiento}

Se invitó al colegio seleccionado a participar de una investigación sobre la crianza. Luego de coordinaciones 
Análisis factorial confirmatorio de la Escala de Estilos de Crianza de Steinberg

pertinentes, se presentó el material a los alumnos(as) y se procedió a explicar el motivo de la investigación. Luego se les dio ejemplos de cómo responder a la prueba y se repartió el material. Los examinadores circulaban por el aula resolviendo cualquier duda. Debido a la escasa experiencia en responder cuestionarios de este tipo, los examinadores pusieron especial cuidado en las instrucciones para responder al instrumento.

Los análisis estadísticos del presente estudio se centraron en la validez y la confiabilidad. Luego de establecidas las propiedades psicométricas del instrumento, se examinaron descriptivamente los datos, tomando en cuenta la probable variabilidad de los resultados como una función del género de los alumnos.

Debido que se tiene una configuración teórica ya definida, se eligió el enfoque confirmatorio del análisis factorial mediante el análisis factorial de grupo múltiple (Merino \& Arndt, en prensa; Nunnally \& Bernstein, 1995). En esta técnica, la extracción de las variables y la estructura interna de cada factor es definida previamente por el investigador, utilizando una matriz binaria de 1 y 0 , los cuales describen la ubicación de los ítems en sus subescalas. Este método es práctico y directo, y con menos presupuestos estadísticos para su aplicación (Merino \& Arndt, en prensa). Para examinar el grado de ajuste del modelo, se comparó la varianza extraída por el modelo propuesto contra los modelos alternativos. Siguiendo las líneas del trabajo de Bernstein (Bernstein \& Teng, 1989; Bernstein, Ellason, Ross \& Vanderlinden, 2001), estos modelos alternativos se construyen a partir de las propiedades distribucionales, la frecuencia media y la variabilidad de las respuestas a los ítems. La hipótesis que se prueba aquí es que la organización de los ítems en sus subescalas proviene de las relaciones conceptuales y en menor grado de los problemas de factorizar ítems categóricos (Merino \& Arndt, en prensa).

La cantidad de respuestas en cada ítem no ha reflejado la muestra total, lo que causará que el tamaño muestral en las submuestras varíe. Esto se observará también en la descripción 
porcentual de los patrones de crianza. Aunque un reemplazamiento estimativo de las respuestas a los ítems hubiera sido una alternativa aceptable, se prefirió mantener intacta esta situación en el cálculo de la información descriptiva para no sobreestimarla y/o subestimarla.

\section{Resultados}

\section{Estructura interna}

El agrupamiento de los ítems para el primer componente es claro y corresponde a la subescala Compromiso. Luego, para el segundo componente, se tiene una carga patrón promedio de 0.45 y claramente refleja a la subescala Autonomía Psicológica. Se debe notar que un ítem (12: "Mis padres me dejan hacer mis propios planes y decisiones para las cosas que quiero hacer") no se ajusta satisfactoriamente.

Originalmente, este ítem fue el único de su subescala que permaneció sin recodificar $y$, al parecer, esto ha influenciado espúreamente en estos resultados; por lo tanto, el ítem 12 muestra un estatus problemático en su ajuste a su componente y al modelo en general.

Finalmente, para el tercer componente, corresponde a la subescala Control Conductual/Supervisión, cuyas cargas son moderadamente aceptables. La estructura factorial de la escala ha sido consistente con lo esperado, confirmando la existencia de tres subescalas. Las cargas estructurales (correlaciones entre ítems y componentes) y de patrón (coeficientes para predecir los componentes por medio de sus ítems) han sido moderadamente altas para los ítems en sus subescalas, y bajas o cercanas a cero para los ítems con las escalas no relacionadas. Sólo un ítem (12) no cargó satisfactoriamente ninguna de las subescalas. Por otro lado, las subescalas tienen inter-correlaciones bajas y demuestran 
Análisis factorial confirmatorio de la Escala de Estilos de Crianza de Steinberg

\section{Cuadro 4}

Matriz de estructura factorial y patrón factorial (entre paréntesis)

\begin{tabular}{|c|c|c|c|c|c|}
\hline Items & $\begin{array}{c}I \\
\text { Compromiso }\end{array}$ & $\begin{array}{c}\text { II } \\
\text { Autonomía } \\
\text { Psicológica }\end{array}$ & $\begin{array}{c}\text { III } \\
\text { Control } \\
\text { Conductual } \\
\text { Supervisión }\end{array}$ & $h^{2}$ & $I S F$ \\
\hline P1 & $0.56(0.56)$ & & & 0.31 & 0.99 \\
\hline P3 & $0.58(0.54)$ & & & 0.35 & 0.93 \\
\hline P5 & $0.44(0.43)$ & & & 0.20 & 0.94 \\
\hline P7 & $0.56(0.54)$ & & & 0.32 & 0.96 \\
\hline P9 & $0.56(0.58)$ & & & 0.32 & 0.97 \\
\hline P11 & $0.65(0.65)$ & & & 0.43 & 0.98 \\
\hline P13 & $0.46(0.45)$ & & & 0.22 & 0.97 \\
\hline P15 & $0.63(0.64)$ & & & 0.41 & 0.97 \\
\hline P17 & $0.66(0.70)$ & & & 0.45 & 0.95 \\
\hline $\mathrm{P} 2$ & & $0.50(0.49)$ & & 0.29 & 0.78 \\
\hline P4 & & $0.34(0.33)$ & & 0.15 & 0.63 \\
\hline P6 & & $0.56(0.56)$ & & 0.33 & 0.91 \\
\hline P8 & & $0.63(0.62)$ & & 0.39 & 0.99 \\
\hline P10 & & $0.43(0.42)$ & & 0.19 & 0.92 \\
\hline P12 & & $0.11(0.12)$ & & 0.07 & 0.38 \\
\hline P14 & & $0.55(0.55)$ & & 0.30 & 0.99 \\
\hline P16 & & $0.50(0.50)$ & & 0.26 & 0.93 \\
\hline P18 & & $0.49(0.48)$ & & 0.24 & 0.94 \\
\hline P19 & & & $0.53(0.56)$ & 0.29 & 0.93 \\
\hline P20 & & & $0.40(0.42)$ & 0.17 & 0.92 \\
\hline P2la & & & $0.46(0.44)$ & 0.21 & 0.97 \\
\hline $\mathrm{P} 21 \mathrm{~b}$ & & & $0.64(0.62)$ & 0.42 & 0.98 \\
\hline P21c & & & $0.52(0.54)$ & 0.27 & 0.98 \\
\hline $\mathrm{P} 22 \mathrm{a}$ & -0.33 & & $0.61(0.56)$ & 0.40 & 0.89 \\
\hline $\mathrm{P} 22 \mathrm{~b}$ & & & $0.66(0.68)$ & 0.45 & 0.98 \\
\hline P22c & & & $0.63(0.63)$ & 0.40 & 0.99 \\
\hline \multicolumn{6}{|c|}{ Correlaciones* } \\
\hline I & 1.0 & -0.09 & -0.31 & & \\
\hline II & -0.04 & 1.0 & -0.04 & & \\
\hline III & -0.31 & -0.02 & 1.0 & & \\
\hline
\end{tabular}

*Valores debajo de la diagonal: correlaciones con todos los ítems incluidos; valores por arriba de la diagonal, sin el ítem 12 en Compromiso. 
que son suficientemente independientes entre ellas. La correlación moderada baja y negativa entre Compromiso y Control/Supervisión indica una ligera relación que es teóricamente esperable.

\section{Cuadro 5}

Comparación y ranking de la varianza explicada por el modelo propuesto y los modelos alternativos

\begin{tabular}{cccc}
\hline & $\begin{array}{c}\text { Varianza } \\
\text { explicada por el } \\
\text { modelo }\end{array}$ & $\begin{array}{c}\text { Raíz Media } \\
\text { Cuadrática } \\
\text { Residual (RMS) }\end{array}$ & Ranking \\
\hline $\begin{array}{c}\text { Modelo } \\
\text { propuesto } \\
\text { completo } \\
\text { Modelo sin } \\
\text { item 12 }\end{array}$ & $30.6 \%$ & .20 & 2 \\
$\begin{array}{c}\text { Modelo } \\
\text { de medias } \\
\text { Desviación } \\
\text { estándar }\end{array}$ & $32.0 \%$ & 0.1 & 1 \\
Simetría & $27.0 \%$ & .22 & 3 \\
\hline
\end{tabular}

En el Cuadro 5, si se considera el porcentaje de varianza explicada, el modelo teórico (Compromiso, Autonomía y Control/ Supervisión) explicó un mayor porcentaje del contenido de los ítems: $30 \%$ y $32 \%$ para el modelo completo y el modelo sin el ítem 12 respectivamente. También, el modelo teórico produjo un mejor ajuste de las correlaciones entre los ítems (raíz media cuadrática residual) y retuvo un mayor porcentaje de varianza inicial que los restantes modelos. Se observó que el modelo propuesto presenta mejores propiedades si el ítem 12 es removido. Las ventajas del modelo teórico sobre los otros modelos, sin embargo, no difieren grandemente, especialmente con el modelo 
construido de las medias de los ítems. Esto señala que el contenido y las medias de los ítems podrían ambos estar influenciando en la formación de las tres escalas, aunque con una mayor influencia del primero.

\section{Confiabilidad}

Mediante el método del coeficiente alfa de Cronbach (Cronbach, 1951) los puntajes de las subescalas arrojaron coeficientes que van desde los marginalmente aceptables a moderadamente bajos. Estos valores se muestran en el Cuadro 6, en el cual también se exhiben los coeficientes alfa del estudio de Mantizicopoulus y Oh-Wang (1998) en adolescentes norteamericanos y coreanos. La probabilidad global sobre las diferencias en la confiabilidad (Merino \& Lautenschlager, 2003) fueron como sigue: $p<0.0001$ en Compromiso, $p=0.033$ en Autonomía Psicológica y $\mathrm{p}=.2768$ en Control Conductual/ Supervisión.

\section{Cuadro 6}

Reporte y comparación de la confiabilidad alfa de Cronbach

\begin{tabular}{|c|c|c|c|c|}
\hline & \multirow{2}{*}{$\begin{array}{c}\text { Mantzicopoulus y } \\
\text { Oh-Wang (1998) } \\
\text { Muestra: } \\
\text { USA } \\
\text { (Coreana) }\end{array}$} & \multicolumn{3}{|c|}{ Presente estudio } \\
\hline & & Total & $\begin{array}{l}\text { Varones } \\
(n=140)\end{array}$ & $\begin{array}{l}\text { Mujeres } \\
(n=63)\end{array}$ \\
\hline Compromiso & $\begin{array}{l}.82 * * \\
(.66)^{*}\end{array}$ & .74 & .74 & .74 \\
\hline Autonomía Psicológi & $\begin{array}{l}c a .73 * \\
(.72)^{*}\end{array}$ & $\begin{array}{l}.62 \\
(.56)\end{array}$ & $\begin{array}{l}.62 \\
(.57)\end{array}$ & $\begin{array}{l}.63 \\
(.53)\end{array}$ \\
\hline Control Conductual & $\begin{array}{l}.69 \\
(.61)\end{array}$ & .66 & .62 & .70 \\
\hline
\end{tabular}

${ }^{* *} \mathrm{p}<.01 ; * \mathrm{p}<.05$ 
Los niveles de confiabilidad interna han tendido a ser, para los puntajes derivados de la muestra de adolescentes peruanos, relativamente bajos en comparación con los adolescentes para las subescalas Compromiso y Autonomía Psicológica, pero no significativamente diferentes en Control. Esto nos advierte del importante impacto de factores de error de medición que subestimaron las estimaciones de confiabilidad. Pero se ha repetido parcialmente el patrón de confiabilidad en las muestras extranjeras: la subescala Compromiso ofrece puntajes con mayor confiabilidad.

\section{Resultados descriptivos}

En el Cuadro 6 se reportan los estadísticos básicos (tendencia central, dispersión y de distribución) para las subescalas Compromiso, Autonomía Psicológica y Control/Supervisión Conductual. Seguidamente, el Cuadro 8 presenta el porcentaje de estilos de crianza en los participantes del estudio. Como se observa ahí, no hay una relación estadísticamente significativa entre el género de los adolescentes y la crianza reportada, $\mathrm{P}^{2}(4$, $152)=9.036, \mathrm{p}>.05 ;$ tampoco se halló una relación estadísticamente significativa entre los estilos de crianza y la edad, $\mathrm{P}^{2}(4,152)=5.529, \mathrm{p}>.05$. Estas variables, por lo tanto, no contribuyen grandemente a explicar las posibles diferencias entre los patrones de crianza, al menos en un nivel estadístico.

Aunque las diferencias porcentuales de los estilos de crianza debido al sexo y la edad no han probado ser significativamente estadísticas, hay diferencias en algunas celdas que son llamativas. Del Cuadro 8 se puede observar para la muestra total que los dos patrones de crianza más frecuentes son de tipo autoritario y permisivo/negligente (33.1\% y $30.5 \%$, respectivamente). Respecto al sexo de los adolescentes, ellos perciben que sus padres tienden a ser más negligentes hacia los varones que hacia las mujeres ( $30.1 \%$ vs. $10.2 \%$ ) y más autoritarios hacia las mujeres que hacia los varones $(48.8 \%$ vs. 28.2$)$. Finalmente, hay una mayor diferencia 
Análisis factorial confirmatorio de la Escala de Estilos de Crianza de Steinberg

entre las edades 11-14 años vs. 15-19 años que reciben una crianza de tipo autoritario ( $40.5 \%$ vs $26.3 \%$, respectivamente).

\section{Cuadro 7}

Estadísticos básicos para Compromiso, Autonomía Psicológica y Control/ Supervisión Conductual

\begin{tabular}{|c|c|c|c|c|c|c|c|c|}
\hline & $n$ & $M$ & $M d$ & $D E$ & $\begin{array}{c}\text { As } \\
\text { (e.e.) }\end{array}$ & $\begin{array}{c}C \\
\text { (e.e.) }\end{array}$ & Min. & Máx. \\
\hline Compromiso & 205 & 15.36 & 14.00 & 4.793 & $\begin{array}{c}.872 \\
(0.170)\end{array}$ & $\begin{array}{c}.413 \\
(0.338)\end{array}$ & 9 & 31 \\
\hline Autonomía & & & & & & & & \\
\hline Psicológica & 213 & 18.92 & 19.00 & 3.840 & .028 & $\begin{array}{c}-.196 \\
(0.167)\end{array}$ & $\begin{array}{c}9 \\
(0.332)\end{array}$ & 28 \\
\hline $\begin{array}{l}\text { Controly } \\
\text { Supervisión } \\
\text { Conductual }\end{array}$ & 209 & 19.44 & 20.00 & 3.430 & $\begin{array}{c}-.557 \\
(0.168)\end{array}$ & $\begin{array}{c}.387 \\
(0.335)\end{array}$ & 8 & 26 \\
\hline
\end{tabular}

M: Media; Md: Mediana; DE: Desviación estándar; As: Asimetría; C: Curtosis; e.e.: Error estándar.

\section{Cuadro 8}

Frecuencias de la tipología de crianza en la muestra total estudiada

\begin{tabular}{lcccccc}
\hline & \multirow{2}{*}{ Total } & \multicolumn{2}{c}{ Sexo } & \multicolumn{2}{c}{ Edad } \\
\cline { 7 - 7 } \cline { 6 - 7 } & & Varón & Mujer & II a I4 años 15 a 19 años \\
\hline Padres Autoritativos & $12(7.8 \%)$ & $6(5.8 \%)$ & $6(12.2 \%)$ & $4(5.4 \%)$ & $8(10.0 \%)$ \\
Padres Negligentes & $36(23.4 \%)$ & $31(30.1 \%)$ & $5(10.2 \%)$ & $17(23.0 \%)$ & $19(23.8 \%)$ \\
Padres Autoritarios & $51(33.1 \%)$ & $29(28.2 \%)$ & $20(40.8 \%)$ & $30(40.5 \%)$ & $21(26.3 \%)$ \\
Padres Permisivos/ & & & & & \\
Indulgentes & $47(30.5 \%)$ & $32(31.1 \%)$ & $15(30.6 \%)$ & $19(25.7 \%)$ & $28(35.0 \%)$ \\
Padres Mixtos & $8(5.2 \%)$ & $5(4.9 \%)$ & $3(6.1 \%)$ & $4(5.4 \%)$ & $4(5.0 \%)$ \\
Total & 154 & 103 & 49 & 74 & 80 \\
\hline
\end{tabular}


César Merino Soto y Stephan Arndt

\section{Discusión}

Desde un enfoque analítico confirmatorio, se han obtenido dos hallazgos relevantes. El primero se refiere a las relaciones entre las subescalas, en que la magnitud de sus correlaciones ha sido débil y moderadamente débil, y demuestran la ortogonalidad entre ellas; es decir, pueden dar información no redundante sobre los constructos medidos. El segundo aspecto de nuestros resultados corresponde a la comparación entre modelos. El modelo propuesto (tres dimensiones de la crianza: Compromiso, Autonomía Psicológica y Control/Supervisión Conductual) ha prevalecido moderadamente sobre los otros modelos alternativos, es decir, aquellos construidos desde las propiedades estadísticas de los ítems más que por las relaciones sustantivas entre ellas. El modelo propuesto, por lo tanto, ha probado dar un relativo mejor ajuste a los datos. Interpretado en forma absoluta, el porcentaje explicado por el modelo ha sido, sin embargo, marginalmente aceptable (30\% de la varianza inicial), pero se elevó ligeramente a $32 \%$ de la varianza explicada al remover un ítem problemático. En términos de la raíz media cuadrática (RMS), el modelo propuesto ha producido las menores correlaciones residuales frente a los modelos alternativos.

Comparativamente, el porcentaje de varianza inicial explicada y la RMS han sido moderadamente mejores que los modelos construidos por las medias, varianzas y simetría distribucional. Pero ha surgido una precaución que se debe tomar, pues estas las diferencias solo han favorecido ligeramente a nuestro modelo frente a los modelos restantes. Al intentar explicar la relación entre los ítems, esto puede sugerir que los modelos alternativos no son tan estadísticamente independientes al modelo propuesto. Estas diferencias, sin embargo, son comparables cuantitativamente a los estudios sobre la factorización de variables categóricas (Bernstein, Ellason, Ross \& Vanderlinden, 2001; Bernstein \& Keith, 1991; Nunnally \& Bernstein, 1995). En estos estudios, a menudo las diferencias entre los modelos propuestos y los 
Análisis factorial confirmatorio de la Escala de Estilos de Crianza de Steinberg

modelos alternativos no son de gran magnitud si son medidos por el porcentaje de varianza retenida por ellos.

Con respecto a las tres escalas evaluadas, la réplica más que la invarianza del modelo factorial propuesto (las tres dimensiones de crianza en adolescentes, de L. Steinberg), ha sido preliminarmente garantizada con los presentes hallazgos. Con el método confirmatorio del análisis de grupo múltiple y rotación oblicua, se ha logrado replicar tres factores, que emergen de una solución de componentes principales. La réplica es el enfoque que puede explicar mejor las variaciones de los componentes en muestras nuevas (Worrell, Vandiver \& Watkins, 2001). Recordemos que el presente estudio se ejecutó en un solo colegio y, como lo refieren Worrell et al. (2001), las variaciones en la estructura interna bajo análisis factorial pueden haber surgido por las particularidades de esta muestra, mientras se esperaría que la estructura de la prueba permanezca estable.

La experiencia de los autores con respecto a la aplicación y análisis de medidas de crianza en el contexto peruano, y la revisión de la literatura relevante, señalan que generalmente se encuentran niveles de confiabilidad moderados e incluso bajos, tal como lo señalarían las convenciones psicométricas. Al parecer, la medición de constructos relacionados con los estilos de crianza tiende a representar significados de amplio rango o de niveles abstractos que los reactivos no pueden capturar claramente. Como lo señalaron Darling y Toyokawa (1997), la distinción entre prácticas de crianza y estilos de crianza se hace prominente en las diferencias de los ítems, unos más conductuales que otros. Si se analiza esta situación desde el lado de los participantes del estudio, es posible pensar que un alto porcentaje de estudiantes de la muestra tenga dificultades para responder a los ítems debido a un bajo nivel de lectura, o de capacidad introspectiva. Estos dos posibles problemas pueden generar actitudes de rechazo, incomodidad y desinterés al completar el cuestionario. Todo esto nos indicaría que su impacto sobre los instrumentos de medición psicológica es directo, como se demostró en el estudio de la 
confiabilidad interna. Esto estaría de acuerdo con la literatura psicométrica respecto a los factores que influencian en la confiabilidad (Brown, 1980; Onwuegbuzie \& Daniel, 2000).

Las diferencias observadas en las confiabilidades desde el enfoque en la prueba de hipótesis (Merino \& Launtechlager, 2003) apuntan a la presencia del sesgo en la medición proveniente de las subescalas (Reynolds, 1982). En este sentido, Reynolds afirma que las diferencias en el nivel estadístico dan evidencias del error de medición debido a que el mismo instrumento mediría, tal vez, diferentes aspectos del constructo en ambos grupos. El análisis de los factores que aportaron al incremento de la varianza irrelevante al constructo (Downing, 2002) señala más bien que los errores de medición provienen predominantemente de las condiciones de aplicación de la prueba, así que damos una menor importancia al impacto producido por un probable funcionamiento diferencial de los ítems. Downing (2002) señala varias fuentes que originan una distorsión de los resultados por el impacto de factores no asociados al objetivo de medición; uno de los mayores factores que recalca es la administración de la prueba.

Se han obtenido evidencias preliminares de la validez de constructo de las dimensiones de crianza medidas por la Escala de Estilos de Crianza de L. Steinberg. Los resultados han provenido de la validez factorial de las tres subescalas: Compromiso, Autonomía Psicológica y Control/Supervisión Conductual. Estas evidencias, sin embargo, solo han sido aceptables. Aunque la estructura interna del instrumento ha provenido esencialmente de las correlaciones y el contenido de los ítems, tiende a confundirse con la influencia de la frecuencia promedio de los ítems (estructura interna proveniente del ordenamiento de los promedios de los ítems).

La confirmación de las tres dimensiones de la crianza, tal como son medidas por las subescalas, nos da indicios de la constancia intercultural de las dimensiones y estilos de crianza construidos sobre estas dimensiones. Este hecho facilitaría la 
Análisis factorial confirmatorio de la Escala de Estilos de Crianza de Steinberg

comparación de las investigaciones sobre la crianza, pues, al utilizarse un mismo instrumento, se reduce la varianza debido al método, es decir, de las diferencias producidas por la aplicación de métodos o instrumentos distintos en la recolección de datos.

Respecto a la confiabilidad por consistencia interna de los puntajes, han sido aceptables para Compromiso y Control/ Supervisión, pero marginalmente aceptable para Autonomía Psicológica. Estos resultados señalan el mayor impacto que ha tenido la varianza irrelevante al constructo, originado aparentemente por las condiciones de evaluación.

El patrón de confiabilidad por consistencia interna ha sido similar a lo reportado por la literatura sobre la medición de las dimensiones de crianza desde los reportes de los hijos, en que éstos tienden a dar resultados moderadamente confiables.

En la muestra estudiada, predominantemente de nivel socioeconómico bajo, se ha hallado una mayor proporción de estilos de crianza permisivo/indulgente y autoritario. Cercanamente, estos hallazgos son consistentes con las investigaciones que reportan la relación entre esta variable demográfica y los patrones de crianza (Majluf, 1989; Shade \& Rojas, 1989). En conjunto, estos patrones pueden ser considerados factores de riesgo que consistentemente están presentes en niveles socioeconómicos en que predomina la pobreza y déficit en la socialización de los niños y adolescentes. Nuestro estudio, en este sentido, proporciona evidencias preliminares sobre la relación entre nivel socioeconómico y estilos de crianza, utilizando un método de auto-reporte aplicado a adolescentes. Cabe señalar que, en el presente estudio, el nivel socioeconómico fue estimado por la pertenencia de los adolescentes a un centro educativo estatal, en una zona urbana en que predominantemente reciben a alumnos con bajo nivel socioeconómico. Es pertinente señalar que no podemos hacer generalizaciones seguras sobre la prevalencia de los estilos de crianza más allá de la muestra estudiada, debido al método de obtención de la muestra. Además, no se ha comparado esta muestra de adolescentes con otros de distinto 
nivel socioeconómico. Finalmente, es posible que las interpretaciones de los adolescentes de las conductas parentales, operacionalizadas con medidas de auto-reporte, tiendan a estar influenciadas por el contexto socio-ambiental en que estas conductas ocurren. Se ha demostrado que el ambiente social inmediato puede alterar el significado y las metas de los procesos familiares (Darling \& Steinberg, 1993). Como señalan Darling y Steinberg (1993), el nivel de riesgo psicosocial proveniente del ambiente es un moderador importante de cómo son percibidas las estrategias educativas y disciplinarias de los padres por parte de los adolescentes.

La presente investigación presenta hallazgos preliminares sobre las propiedades psicométricas del instrumento; y, debido a la magnitud de los estadísticos hallados y al diseño muestral, se requieren estudios adicionales para obtener evidencias que completen la información de la validez y confiabilidad.

\section{Referencias}

American Psychiatric Association. (1994). Diagnostic and statistical manual of mental disorders (4ta. ed.). Washington, DC, EE. UU.: APA.

Arrindel, W. A., Ritcher, J., Eisemann, M., Gärling, T., Ryde’n, O., Hansson, S. B. et al. (2001). The short-EMBU in East-Germany and Sweden: A cross-national factorial validity extension. Scandinavian Journal of Psychology, 42(2), 157-160.

Baumrind, D. (1991). Parenting styles and adolescent development. En J. Brooks-Gunn, R. Lerner \& A. C. Petersen (Eds.), The encyclopedia of adolescence (pp. 746-758). Nueva York: Garland.

Bernstein, I. H. \& Keith, J. B. (1991). Reexamination of Eisen, Zellman, and McAlister's health belief model questionnaire. Health Education Quarterly, 18(2), 207-220. 
Análisis factorial confirmatorio de la Escala de Estilos de Crianza de Steinberg

Bernstein, I. H., Ellason, J. W., Ross, C. A. \& Vanderlinden, J. (2001). On the dimensionalities of the Dissociative Experiences Scale (DES) and the Dissociation Questionnaire. Journal of Trauma \& Dissociation, 2(3), 103-122.

Bernstein, I. H., Jaremko, M. E. \& Hinkley, B. S. (1994). On the utility of the SCL-90 with low-back pain patients. Spine, $19(1), 42-48$.

Bernstein, I. H. \& Teng, G. (1989). Factoring items and factoring scales are different: Spurious evidence for multidimensionality due to item categorization. Psychological Bulletin, 105(3), 467477.

Brown, F. G. (1980). Principios de la medición en psicología y educación. México: Manual Moderno.

Buri, J. (1991). Parental Authority Questionnaire. Journal of Personality Assessment, 51(1), 110-119.

Cronbach, L. J. (1951). Coefficient alpha and the internal structure of tests. Psychometrika, 16(3), 297-334.

Cronbach, L. J. \& Meehl, P. E. (1955). Construct validity in psychological tests. Psychological Bulletin, 52, 281-302.

Darling, N. (1999, marzo). Parenting style and its correlates. ERIC Digest EDO-PS-99-3.

Darling, N. \& Steinberg, L. (1993). Parenting style as context: An integrative model. Psychological Bulletin, 113(3), 1-10.

Darling, N. \& Toyokawa, C. (1997). Construction of the Parenting Style Inventory (PSI-II): Revised Edition. Documento no publicado, Pennsylvania State University, EE. UU.

Devereux, E. D., Bronfenbrenner, U. \& Rodgers, R. R. (1969). Child-rearing in England and the United States: A crossnational comparison. Journal of Marriage and the Family, 5, 257-270.

Downing, S. M. (2002). Threats to the validity of locally developed multiple-choice tests in medical education: Construct-irrelevant variance and construct underrepresentation. Advances in Health Sciences Education, 7, 235-241. 
Enzmann, D. (1997). RanEigen: A program to determine the parallel analysis criterion for the number of principal components. Applied Psychological Measurement, 21, 232.

Fleming, J. S. (2003). Computing measures of simplicity of fit for loadings in factor analytically derived scales. Behavior Research Methods, Instruments, and Computers, 35(4), 520524.

Gregory, R. J. (2000). Psychological testing: History, principles, and applications (3ra. ed.). Boston: Allyn \& Bacon.

Lamborn, S. D., Mounts, N., Steinberg, L. \& Dornbusch, S. (1991). Patterns of competence and adjustment among adolescents from authoritative, authoritarian, indulgent and neglectful families. Child Development, 62, 1049-1065.

Lautenschlager, G. J. (1989). ALPHATST: Testing for differences in values of coefficient alpha. Applied Psychological Measurement, 13, 284.

Lieb, R., Isensee, B., Höfler, M., Pfister, H. \& Wittchen, H. U. (2002). Parental major depression and the risk of depression and other mental disorders in offspring: A prospectivelongitudinal community study. Archives of General Psychiatry, 59, 365-374.

Lieb, R., Merikangas, K. R., Höfler, M., Pfister, H., Isensee, B. \& Wittchen, H. U. (2002). Parental alcohol use disorders and alcohol use and disorders in offspring: A community study. Psychosocial Medicine, 32, 63-78.

Lieb, R., Wittchen, H., Höfler, H., Fuetsch, M., Stein, M. B. \& Merikangas, K. R. (2000). Parental psychopathology, parenting styles, and the risk of social phobia in offspring: A prospective-longitudinal community study. Archives of General Psychiatry, 57, 859-866.

Maccoby, E. E. \& Martin, J. (1983). Socialization in the context of the family: Parent-child interaction. En P. H. Mussen (Series Ed.) \& E. M. Hetherington (Vol. Ed.), Handbook of child psychology: Vol. 4. Socialization, personality, and social development (4a. ed., pp. 1-101). Nueva York: Wiley. 
Análisis factorial confirmatorio de la Escala de Estilos de Crianza de Steinberg

Majluf, A. (1989). Prácticas de crianza en madres de estratos medio y bajo de Lima. Revista de Psicología de la Pontificia Universidad Católica del Perú, 7(2), 151-161.

Mantzicopoulus, P. Y. \& Oh-Hwang, Y. (1998). The relationship of psychosocial maturity to parenting quality and intellectual ability for American and Korean adolescents. Contemporary Educational Psychology, 23, 195-206.

Martin, J. D. \& Gray, L. N. (1971). Measurement of relative variation: Sociological examples. American Sociological Review, 36(3), 496-502.

Merino, C. \& Arndt, S. (en prensa). Un enfoque confirmatorio para el análisis de escalas: análisis factorial de grupo múltiple. Revista de Psicología de la Universidad Inca Garcilaso de la Vega.

Merino, C., Cohen, B. \& Díaz, M. (2003). De los niños a los padres: el Inventario de Percepción de Conductas Parentales. Persona, 6, 135-150.

Merino, C. \& Lautenschlager, G. (2003). Comparación estadística de la confiabilidad alfa de Cronbach: aplicaciones en la medición educacional. Revista de Psicología de la Universidad de Chile, 12(2), 129-139.

Navas, M. (2001). La fiabilidad como criterio métrico de la calificación global del test. En M. L. Navas (Ed.), Métodos, diseños y técnicas de investigación psicológica (pp. 213-262). Madrid: UNED.

Nunnally, J. C. \& Bernstein, I. J. (1995). Teoría psicométrica (3ra. ed.). México: McGraw Hill.

Onwuegbuzie, A. A. \& Daniel, L. G. (2000, noviembre). Reliability generalization: The importance of considering sample specificity, confidence intervals, and subgroup differences. Documento presentado en la reunión anual de la Mid-South Educational Research Association, Bowling Green, KY, EE. UU.

Perris, C., Jacobsson, L., Lindström, H., von Knorring, L. \& Perris, H. (1980). Development of an inventory for assessing 
memories of parental rearing behaviour. Acta Psychiatrica Scandinavica, 61, 265-274.

Reynolds, C. R. (1982). Construct and predictive bias. En R. A. Berk, Handbook of methods for detecting test bias (pp. 199227). Baltimore, MD: Johns Hopkins University Press.

Robinson, C. C., Mandleco, B., Olsen, S. F. \& Hart, C. H. (1995). Authoritative, authoritarian, and permissive parenting practices: Development of a new measure. Psychological Reports, 77, 819-830.

Schade, B. \& Rojas, C. (1989). Niños en extrema pobreza... ¿Socialización deficitaria?: resultado de un estudio empírico en un pueblo joven de Lima. Revista de Psicología de la Pontificia Universidad Católica del Perú, 7(2), 139-150.

Schluderman, E. H. \& Schluderman, S. H. (1988). Children's report on parent behavior (CRPBI-108, CRPBI-30) for older children and adolescents (Tech. Rep.). Documento no publicado, Universidad de Manitoba, Winnipeg, MB, Canadá. Steinberg, L., Elman, J. D. \& Mounts, N. S. (1989). Authoritative parenting, psychosocial maturity, and academic success among adolescents. Child Development, 60, 1424-1436.

Steinberg, L., Lamborn, S., Dornbusch, S. \& Darling, N. (1992). Impact of parenting practices on adolescent achievement: Authoritative parenting, school involvement, encouragement to succeed. Child Development, 63, 1266-1281.

Worrell, F. C., Vandiver, B. J. \& Watkins, M. W. (2001). Construct validity of the Learning Behavior Scale with an independent sample of students. Psychology in Schools, 38(3), 207-215. 
Análisis factorial confirmatorio de la Escala de Estilos de Crianza de Steinberg

\section{Anexo}

Mis padres

Por favor, responde a TODAS las siguientes preguntas sobre los padres (o apoderados) con los que tú vives. Si pasas más tiempo en una casa que en otra, responde las preguntas sobre las personas que te conocen mejor. Es importante que seas sincero.

Si estás MUY DE ACUERDO haz una $X$ sobre la raya en la columna (MA) Si estás ALGO DE ACUERDO haz una $X$ sobre la raya en la columna (AA) Si estás ALGO EN DESACUERDO haz una $X$ sobre la raya en la columna (AD)

Si estás MUY EN DESACUERDO haz una X sobre la raya en la columna (MD)

MA AA AD MD

1. Puedo contar con la ayuda de mis padres si tengo algún tipo de problema.

2. Mis padres dicen o piensan que uno no debería discutir con los adultos.

3. Mis padres me animan para que haga lo mejor que pueda en las cosas que yo haga.

4. Mis padres dicen que uno debería no seguir discutiendo y ceder, en vez de hacer que la gente se moleste con uno

5. Mis padres me animan para que piense por mí mismo. - _ _

6. Cuando saco una baja nota en el colegio, mis padres me hacen la vida "difícil".

7. Mis padres me ayudan con mis tareas escolares si hay algo que no entiendo.

8. Mis padres me dicen que sus ideas son correctas y que yo no debería contradecirlas.

9. Cuando mis padres quieren que haga algo, me explican por qué.

10. Siempre que discuto con mis padres, me dicen cosas como, "Lo comprenderás mejor cuando seas mayor"

11. Cuando saco una baja nota en el colegio, mis padres me animan a tratar de esforzarme.

12. Mis padres me dejan hacer mis propios planes y decisiones para las cosas que quiero hacer.

13. Mis padres conocen quiénes son mis amigos. 
14. Mis padres actúan de una manera fría y poco amigable si yo hago algo que no les gusta.

15. Mis padres dan de su tiempo para hablar conmigo. .. - $\quad$ - $\quad-$

16. Cuando saco una baja nota en el colegio, mis padres me hacen sentir culpable

17. En mi familia hacemos cosas para divertimos o pasarla bien juntos.

18. Mis padres no me dejan hacer algo o estar con ellos cuando hago algo que a ellos no les gusta

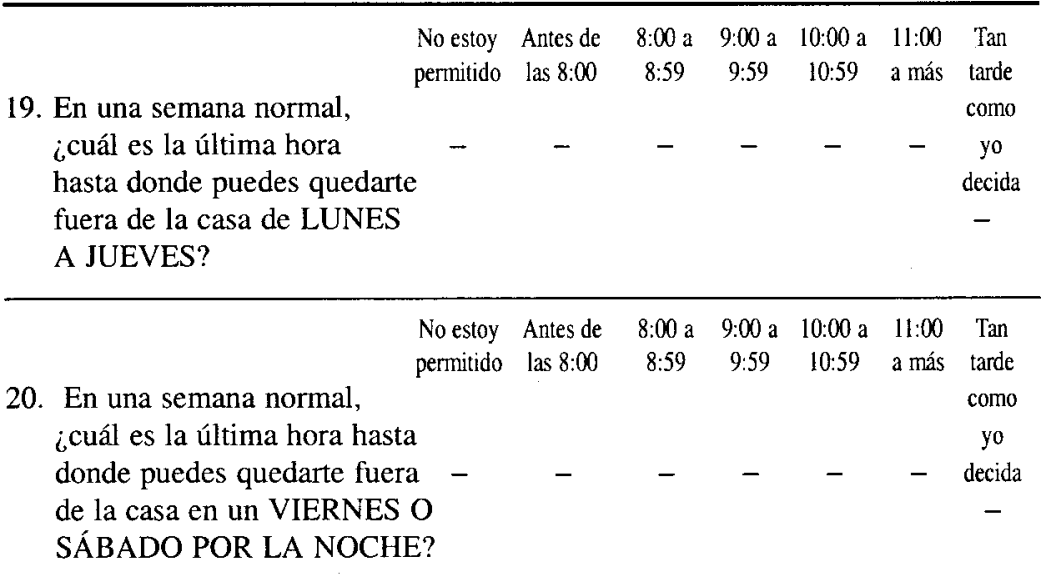

21. ¿Qué tanto tus padres TRATAN No tratan Tratan un poco Tratan mucho de saber.
a. Dónde vas en la noche?
b. Lo que haces con tu tiempo libre? ..
c. Dónde estás mayormente en las tardes después del colegio?

22. ¿Qué tanto tus padres REALMENTE . No saben Saben un poco Saben mucho saben....
a. Dónde vas en la noche?
b. Lo que haces con tu tiempo libre?..
c. Dónde estás mayormente en las tardes después del colegio?

$\begin{array}{lll}- & - & - \\ - & - & - \\ - & - & -\end{array}$

